\section{Período de adaptação a dietas com baixa ou alta fibra sobre a digestibilidade e as características fecais em cães}

\author{
[Adaptation period to diets with low or high fiber on digetibility and faecal \\ characteristics in dogs] \\ T. Ramos ${ }^{1}$, G.C.B. Kaelle ${ }^{2}$, D.C. Lima ${ }^{2}$, M. Scheraiber ${ }^{2}$, S.G. Oliveira ${ }^{1}$, A.P. Félix ${ }^{1}$ \\ ${ }^{1}$ Universidade Federal do Paraná - Curitiba, PR \\ ${ }^{2}$ Aluno de pós-graduação - Universidade Federal do Paraná - Curitiba, PR
}

T. Ramos ${ }^{1}$
https://orcid.org/0000-0003-0752-2373
G.C.B. Kaelle*,
https://orcid.org/0000-0003-0171-741X
D.C. Lima ${ }^{2}$,
https://orcid.org/0000-0001-9677-7436
M. Scheraiber ${ }^{2}$,
https://orcid.org/0000-0003-0370-4778
S. G. Oliveira1,
https://orcid.org/0000-0002-2913-1173
A.P. Félix1
https://orcid.org/0000-0002-8570-5725

\title{
RESUMO
}

Objetivou-se avaliar três períodos de adaptação, cinco, 10 e 15 dias, a dietas com baixa ou alta fibra sobre a digestibilidade e características fecais. Foram utilizadas duas dietas, uma contendo $0 \%$ de casca de soja $(0 \% \mathrm{CS})$ e outra com $15 \%$ de casca de soja $(15 \% \mathrm{CS})$, fornecidas a 12 cães adultos. Ao final de cada período, houve coleta total de fezes para avaliação dos coeficientes de digestibilidade aparente (CDA), energia metabolizável (EM) e características fecais. Além disso, foi avaliada a palatabilidade das dietas. Como resultado, não houve efeito do período de adaptação sobre a digestibilidade das dietas $(\mathrm{P}>0,05)$. A dieta $15 \% \mathrm{CS}$ apresentou menor CDA da matéria seca (MS) e EM, em relação à dieta $0 \% \mathrm{CS}(\mathrm{P}<0,05)$. A consistência fecal dos cães não diferiu entre as dietas e os períodos $(\mathrm{P}>0,05)$. Houve redução no $\mathrm{pH}$ e na amônia fecal dos cães alimentados com a dieta 15\%CS (P<0,05), após 10 dias de alimentação. Não houve diferença na palatabilidade das dietas $(\mathrm{P}>0,05)$. Períodos de adaptação à dieta superiores a cinco dias não alteram a digestibilidade e a maioria das características fecais de cães, exceto a amônia fecal. A inclusão de $15 \% \mathrm{CS}$ na dieta reduz a digestibilidade da MS e a EM, bem como o pH e a amônia fecal.

Palavras-chave: casca de soja, protocolo experimental, valor nutricional

\begin{abstract}
This study aimed to evaluate three periods of adaptation, 5, 10 and 15 days, to diets with low or high fiber on digestibility and fecal characteristics. Two diets were used, one containing $0 \%(0 \%$ SH) and one containing $15 \%$ soybean hulls (15\% SH). Diets were provided to 12 adult dogs. At the end of each period there was total collection of feces to evaluate the apparent digestibility coefficients (ADC), metabolizable energy (ME) and fecal characteristics. We also evaluated diet palatability. There was no effect of the adaptation period on digestibility of diets $(P>0.05)$. The $15 \%$ SH diet reduced ADC of dry matter $(D M)$ and $M E$, compared to the $0 \%$ SH diet $(P<0.05)$. Fecal consistency of the dogs did not differ among diets and periods $(P>0.05)$. There was a decrease in fecal $\mathrm{pH}$ and ammonia in dogs fed the diet with $15 \% \mathrm{SH}$ $(P<0.05)$ after 10 days of feeding $(P<0.05)$. There was no difference in diet palatability $(P>0.05)$. Diet adaptation longer than 5 days did not alter the digestibility and most fecal characteristics of dogs, except for fecal ammonia. The inclusion of $15 \% \mathrm{SH}$ in diet reduces DM digestibility and fecal pH and ammonia in dogs.
\end{abstract}

Keywords: soybean hulls, experimental protocol, nutritional value

\section{INTRODUÇÃO}

As fontes de fibras são utilizadas em alimentos comerciais para cães com diversas finalidades. Entre elas cabe citar: restringir o consumo, diluir

Recebido em 20 de dezembro de 2017

Aceito em 28 de dezembro de 2018

*Autor para correspondência (corresponding author)

E-mail: gislaine_bill@yahoo.com.br a energia da dieta (Mateos et al., 2012) e reduzir a absorção de glicose, buscando, assim, o controle de peso dos animais, bem como estimular o peristaltismo e a formação do bolo fecal (Nutrient..., 2006). A partir disso, dependendo da quantidade e do tipo de fibra 
fornecida, poderá ocorrer diferentes efeitos sobre a digestibilidade dos nutrientes da dieta (Campbell e Campbell, 2009).

As fibras são carboidratos estruturais, originários principalmente das paredes celulares de vegetais (Koopel et al., 2015), como a celulose, a hemicelulose, as pectinas, as gomas $\mathrm{e}$ as mucilagens, mais a lignina (Guillon e Champ, 2000). Uma fonte de fibra que está sendo avaliada como alternativa na alimentação de cães é a casca de soja (Cole et al., 2009), a qual é obtida durante o processo do grão para obtenção do farelo.

A maioria dos ensaios de digestibilidade realizados em cães utiliza cinco dias para adaptação dos animais às dietas e instalações (Dog..., 2004). Porém, quando são fornecidas dietas contendo altos teores de fibras, é possível que seja necessário maior período de adaptação. Isso se deve à característica das fibras sobre a velocidade de passagem da digesta, as quais podem alterar essa velocidade e causar alterações na mucosa e microbiota intestinal (Nutrient..., 2006), demandando tempo ao organismo para se adaptar a essas alterações.

Com isso, o objetivo do presente estudo foi avaliar o efeito de três períodos de adaptação, sendo eles cinco, 10 e 15 dias, às dietas com baixa ou alta fibra sobre a digestibilidade e as características fecais de cães. Ainda, será avaliada a palatabilidade das dietas.

\section{MATERIAL E MÉTODOS}

O experimento foi aprovado pela Comissão de Ética no Uso de Animais da Universidade Federal do Paraná, sob o protocolo número 024/2015. Foram avaliadas duas dietas, uma controle, sem adição de fonte de fibra $(0 \% \mathrm{CS}) \mathrm{e}$ uma com $15 \%$ de casca de soja $(15 \% \mathrm{CS})$, fornecidas a 12 cães adultos, seis fêmeas e seis machos, da raça Beagle. As dietas foram oferecidas duas vezes ao dia, por um período de adaptação de cinco, 10 e 15 dias, seguidos de cinco dias de coleta total de fezes em cada período, resultando, ao final de cada coleta, em uma mistura composta das fezes de cada animal. Os procedimentos seguiram as recomendações da Association of American Feed Control Officials (Dog..., 2004). Durante o período de coleta, as fezes foram coletadas e pesadas duas vezes por dia e armazenadas em potes plásticos individuais, previamente identificados, tampados e armazenados em freezer a $-21^{\circ} \mathrm{C}$, para posteriores análises.

As dietas foram formuladas para atender as necessidades nutricionais de cães adultos, preconizadas pela AAFCO (Dog..., 2004). Antes do início do ensaio de digestibilidade, as rações foram analisadas quanto à umidade (UM), à proteína bruta $(\mathrm{PB})$, à matéria mineral $(\mathrm{MM})$, ao cálcio $(\mathrm{Ca})$, ao fósforo $(\mathrm{P})$, à fibra bruta $(\mathrm{FB}) \mathrm{e}$ ao extrato etéreo em hidrólise ácida (EEA), de acordo com a AOAC (Official..., 1995), bem como quanto à energia bruta (EB), em bomba calorimétrica. A Tab. 1 apresenta os ingredientes e a composição química das dietas experimentais.

Tabela 1. Ingredientes e composição química analisada das dietas experimentais

\begin{tabular}{lcc}
\multicolumn{1}{c}{ Ingredientes (g/kg) } & $0 \% \mathrm{CS}$ & $\begin{array}{c}15 \% \\
\mathrm{CS}\end{array}$ \\
\hline Milho & 300,0 & 150,0 \\
Gordura de aves & 115,43 & 115,43 \\
Farelo de soja 46\% & 150,0 & 150,0 \\
Glúten de milho 60 & 170,0 & 170,0 \\
Farinha de vísceras de aves & 220,0 & 220,0 \\
Sal branco comum & 5,0 & 5,0 \\
Palatabilizante líquido & 30,0 & 30,0 \\
BHA & 0,075 & 0,075 \\
BHT & 0,15 & 0,15 \\
Ácido cítrico & 0,35 & 0,35 \\
Propionato de cálcio & 2,0 & 2,0 \\
Cloreto de colina & 4,0 & 4,0 \\
Premix & 3,0 & 3,0 \\
Casca de soja & 0 & 150,0 \\
Composição química (g/kg de matéria seca) \\
Matéria seca & 950,4 & 967,6 \\
Proteína bruta & 386,7 & 377,4 \\
Extrato etéreo em hidrólise & 179,4 & 174,2 \\
ácida & 41,1 & 88,6 \\
Fibra bruta & 73,0 & 75,8 \\
Matéria mineral & 8,9 & 7,7 \\
Cálcio & 7,0 & 7,1 \\
Fósforo & 3896,6 & 3689,6 \\
EM ${ }^{*}$ (kcal/kg) & 5 & 3 \\
\hline
\end{tabular}

*1' Energia metabolizável estimada por: EM (kcal.g-1)

$=[(3,5 \times \mathrm{PB} \%+8,5 \times \mathrm{EEA} \%+3,5 \times \mathrm{ENN} \%)] / 100$.

Ao final dos períodos de coleta, a mistura composta de fezes de cada animal em cada período foi descongelada, homogeneizada e 
submetida à secagem em estufa a $55^{\circ} \mathrm{C}$, por 72 horas, e posterior moagem a $1 \mathrm{~mm}$ para realização das análises de UM, PB, EEA e EB. Com base nos resultados obtidos, foram calculados os coeficientes de digestibilidade aparente (CDA) da MS, da PB, do EEA e da EB, segundo a AAFCO (Dog..., 2004). A EM foi estimada segundo a AAFCO (Dog..., 2004), considerando-se o fator de correção para perda energética pela urina.

Durante o período de coleta fecal, foi avaliada a qualidade das fezes frescas pela avaliação da consistência, da produção fecal, do pH (em pHmetro digital), do nitrogênio amoniacal e da matéria seca fecal (MSf). A consistência fecal foi avaliada por meio de um escore com graduação de 1 a 5 , sendo 1 o indicativo de fezes pastosas e sem forma, 2 o indicativo de fezes macias e malformadas, 3 o indicativo de fezes macias, formadas e úmidas, 4 o indicativo de fezes bem formadas e consistentes e 5 o indicativo de fezes bem formadas, duras e secas (Sá-Fortes, 2005).

A palatabilidade das dietas foi determinada pela mensuração da preferência alimentar e da primeira escolha e pela razão de ingestão entre as rações ofertadas aos 12 cães e foi mensurada comparando-se as dietas em pares (Griffin, 2003), resultando no teste: dieta com 0\%CS vs. dieta $15 \%$ CS. O teste foi composto por três dias consecutivos, nos quais foram fornecidos dois potes contendo as duas diferentes dietas a serem comparadas, durante um período de 30 minutos. A quantidade fornecida foi $30 \%$ superior às necessidades de EM preconizadas pelo NRC (Nutrient..., 2006) para cães adultos. As quantidades fornecidas e as sobras foram quantificadas para se calcular a razão da ingestão. Por sua vez, a primeira escolha foi definida pelo registro do primeiro pote de que o animal se aproximou durante a oferta simultânea dos alimentos. A posição dos potes foi alternada entre os dias de avaliação para se evitarem preferências por posição de alimentação. A razão de ingestão foi calculada com base no consumo (fornecido - sobras) relativo das dietas (A e B), sendo: razão de ingestão $=\mathrm{g}$ ingeridas da dieta $\mathrm{A}$ ou $\mathrm{B} / \mathrm{g}$ totais consumidas $(\mathrm{A}+\mathrm{B})$.

O experimento de digestibilidade foi analisado segundo delineamento inteiramente ao acaso, em parcela subdividida no tempo, sendo a parcela as dietas e as subparcelas os tempos de adaptação, totalizando seis repetições por tratamento. Os dados foram submetidos ao teste de Bartlett para verificar a homogeneidade das variâncias. Os dados com variâncias homogêneas foram submetidos à análise de variância e, quando significativos, as médias foram comparadas pelo teste de Tukey, a 5\% de probabilidade. Os dados com variâncias heterogêneas foram analisados pelo teste de Kruskal-Wallis $(\mathrm{P}<0,05)$.

Para o experimento de palatabilidade, o delineamento adotado foi inteiramente ao acaso, totalizando 36 repetições por teste (12 cães x três dias). Os dados de razão de ingestão foram analisados pelo teste t-Student, e a primeira escolha pelo teste qui-quadrado, ambos a $5 \%$ de probabilidade.

\section{RESULTADOS E DISCUSSÃO}

Os resultados dos CDA e da EM das dietas, bem como da MSf, estão apresentados na Tab. 2. A dieta $15 \% \mathrm{CS}$ apresentou menor CDA da MS e da EM, em relação à dieta $0 \% \mathrm{CS} \quad(\mathrm{P}<0,05)$, enquanto os CDA dos demais nutrientes não diferiram entre as dietas $(\mathrm{P}>0,05)$. A mesma situação ocorreu no trabalho de Sabchuk et al. (2017), que utilizaram níveis crescentes de casca de soja na dieta em cães e houve diminuição dos CDA dos nutrientes e da EM, conforme aumentava a quantidade de casca de soja na dieta (inclusão máxima de 16\%). Do mesmo modo, Cole et. al. (1999), ao avaliarem dietas contendo até $9 \%$ de casca de soja em cães, encontraram redução nos CDA da MS, MO, EB e EM. A explicação para esse acontecimento se deve ao fato de a casca de soja ser rica em fibras insolúveis e esse excesso na dieta pode aumentar o peristaltismo, reduzindo o tempo de contato dos nutrientes com as enzimas digestivas. Ainda, pode formar uma barreira física sobre os nutrientes, reduzindo a ação enzimática (Cole $e t$ al., 1999; Nutrient..., 2006).

Não foi observada diferença na digestibilidade e EM das dietas entre os períodos de adaptação avaliados $(\mathrm{P}>0,05)$. Esse mesmo resultado foi encontrado por Nott et al. (1994), que avaliaram a digestibilidade da dieta após quatro, oito ou 15 dias de alimentação em cães. Porém, os autores não avaliaram dietas com alta fibra, ao contrário do que ocorreu no presente trabalho. Já Brunsgaard et al. (1995), ao utilizarem ratos e dietas com diferentes fontes de fibra, observaram 
que a digestibilidade das dietas contendo goma guar e pectina se estabilizou apenas por volta dos 10 dias de alimentação, enquanto a digestibilidade da dieta com baixa fibra ou rica em celulose não foi alterada pelo período de adaptação. Os autores explicam que é provável que fibras solúveis apresentem maior capacidade de alterar o padrão fermentativo do intestino ao longo do tempo, alterando a digestibilidade da dieta. A maior quantidade de fibra insolúvel na casca de soja (relação fibra insolúvel:solúvel = 9:1, Sabchuk et al., 2017) pode explicar o fato de não ter ocorrido diferença na digestibilidade da dieta ao longo do tempo no presente estudo. Assim, é provável que dietas contendo fontes de fibras solúveis necessitem ser fornecidas por maior período de adaptação em cães, merecendo maiores estudos.

Tabela 2. Coeficientes de digestibilidade aparente (CDA, \%) e energia metabolizável (EM, kcal/kg) de dietas contendo $0 \%(0 \% \mathrm{CS})$ e $15 \%(15 \% \mathrm{CS})$ de casca de soja, avaliados em três períodos de adaptação em cães

\begin{tabular}{|c|c|c|c|c|c|c|c|c|c|c|}
\hline Períodos & 5 dias & & 10 dias & & 15 dias & & & & & Valor \\
\hline Dietas & $0 \%$ & $15 \%$ & $0 \%$ & $15 \%$ & $0 \%$ & $15 \%$ & EFIV & P Dieta & Período & DxP \\
\hline MS & $81,0^{\mathrm{a}}$ & $73,7^{b}$ & $80,0^{\mathrm{a}}$ & $75,9^{b}$ & $79,6^{\mathrm{a}}$ & $72,3^{b}$ & 0,807 & $<0,001$ & 0,541 & 0,636 \\
\hline PB & 85,4 & 83,2 & 85,6 & 84,7 & 85,4 & 82,8 & 0,522 & 0,608 & 0,169 & 0,636 \\
\hline EEA & 91,7 & 88,5 & 91,7 & 88,0 & 89,7 & 85,8 & 0,647 & 0,076 & 0,141 & 0,962 \\
\hline EM & $4640,1^{\mathrm{a}}$ & $4074,3^{\mathrm{b}}$ & $4681,0^{\mathrm{a}}$ & $4143,9^{b}$ & $4639,2^{\mathrm{a}}$ & $4113,6^{b}$ & 56,41 & $<0,001$ & 0,334 & 0,881 \\
\hline MSf & 34,1 & 33,3 & 33,5 & 35,8 & 34,4 & 33,8 & 0,744 & 0,875 & 0,855 & 0,597 \\
\hline
\end{tabular}

${ }^{\mathrm{a}, \mathrm{b}}$ Médias seguidas por letras distintas diferem pelo teste de Tukey $(\mathrm{P}<0,05)$.

EPM: erro-padrão da média; MS: matéria seca; PB: proteína bruta; EEA: extrato etéreo em hidrólise ácida; EM: energia metabolizável; MSf: matéria seca fecal (\%).

Para características fecais, não houve efeito da dieta ou do período sobre o escore fecal $(\mathrm{P}>0,05)$. Esses dados corroboram os de Sabchuk et al. (2017), que também não encontraram diferença na consistência fecal de cães alimentados com dietas contendo ou não casca de soja. Já Cole et al. (1999) relataram aumento da consistência fecal com a inclusão de casca de soja na dieta em cães. A divergência de resultados encontrados em relação à consistência fecal de cães alimentados com casca de soja provavelmente se deve à grande variação na relação de fibra insolúvel e fibra solúvel que esse ingrediente pode apresentar, variando de 5:1 a 14,4:1 (Cole et al., 1999).

A produção fecal dos animais não apresentou diferença estatística entre os períodos, mas foi maior nos cães alimentados com a dieta contendo $15 \%$ CS $(\mathrm{P}<0,05)$. Esse fato era esperado, já que foi utilizada, em um dos tratamentos, uma dieta com maior quantidade de fibra insolúvel, que, devido à sua indigestibilidade, aumenta o bolo fecal (Nutrient..., 2006). O mesmo resultado foi observado por Zhang et al. (2013), ao avaliarem dietas contendo 0 a $55 \%$ de polpa de beterraba em leitões, e por Sabchuck et al. (2017), ao avaliarem dietas contendo 0 a $16 \%$ de casca de soja em cães.
Houve redução no $\mathrm{pH}$ fecal com a inclusão de casca de soja na dieta $(\mathrm{P}<0,05)$ em todos os períodos avaliados. Os cães alimentados com a dieta contendo $15 \% \mathrm{CS}$ apresentaram menor teor de amônia fecal que os animais recebendo a dieta controle $(\mathrm{P}<0,05)$. A redução da amônia e do $\mathrm{pH}$ fecal pode indicar que as fibras solúveis da casca de soja foram fermentadas no cólon, acidificando o intestino, por meio da produção de lactato e ácidos graxos de cadeia curta. Desse modo, há redução na produção e volatilização da amônia no intestino (Kuzmuk et al., 2005; Yamka et al., 2006). Esses mesmos resultados foram relatados por Sabchuk et al. (2017) em cães, ao fornecerem dieta contendo até $16 \%$ de casca de soja. A amônia fecal não diferiu entre as dietas após cinco dias de adaptação, no entanto houve diferença após 10 e 15 dias de alimentação $(\mathrm{P}<0,05)$. Esses resultados podem ser observados na Tab. 3.

Com relação ao teste de palatabilidade, não houve diferença na preferência e na primeira escolha das dietas $(\mathrm{P}>0,05)$, como mostra a Tab. 4. A mesma situação ocorreu no trabalho de Sabchuk et al. (2017). Entretanto, Weber et al. (2007) verificaram menor palatabilidade em dietas com alta inclusão de fibras em cães. 
Tabela 3. Características fecais de cães alimentados com dietas contendo 0\% (0\%CS) e 15\% (15\%CS) de casca de soja, avaliados em três períodos de adaptação

\begin{tabular}{cccccccc}
\hline Períodos & 5 dias & \multicolumn{5}{c|}{15 dias } & \multirow{2}{*}{ Valor de P } \\
\hline Dietas & $0 \%$ & $15 \%$ & $0 \%$ & $15 \%$ & $0 \%$ & $15 \%$ & 0,683 \\
EF & 3,9 & 3,8 & 3,8 & 3,8 & 3,8 & 3,9 & 0,001 \\
pH & $6,58^{\mathrm{a}}$ & $6,32^{\mathrm{b}}$ & $6,62^{\mathrm{a}}$ & $6,31^{\mathrm{b}}$ & $6,57^{\mathrm{a}}$ & $6,36^{\mathrm{b}}$ & 0,005 \\
$\mathrm{NH}_{3}$ & $0,10^{\mathrm{ab}}$ & $0,10^{\mathrm{ab}}$ & $0,14^{\mathrm{a}}$ & $0,09^{\mathrm{b}}$ & $0,13^{\mathrm{a}}$ & $0,09^{\mathrm{b}}$ & $<0,001$ \\
$\mathrm{PF}$ & $0,15^{\mathrm{b}}$ & $0,21^{\mathrm{a}}$ & $0,13^{\mathrm{b}}$ & $0,18^{\mathrm{a}}$ & $0,15^{\mathrm{b}}$ & $0,20^{\mathrm{a}}$ & $<0,13$ \\
\hline
\end{tabular}

${ }^{\mathrm{a}, \mathrm{b}}$ Medianas seguidas por letras distintas diferem pelo teste de Kruskal-Wallis $(\mathrm{P}<0,05)$.

EF: escore fecal; NH3: nitrogênio amoniacal (\%); PF: produção fecal (g fezes/g matéria seca ingerida).

Tabela 4. Primeira escolha (n) e razão de ingestão (RI \pm erro-padrão) de cães alimentados com a dieta $0 \%$ de casca de soja (0\%CS) e com dieta contendo $15 \%$ de casca de soja (15\%CS)

\begin{tabular}{ccc}
\hline Dieta AxB & $\mathrm{n}^{\mathrm{a}}$ & RI da dieta $\mathrm{A}^{\mathrm{b}}$ \\
\hline $0 \%$ CS x 15\%CS & 20 & $0,5 \pm 0,05$ \\
\hline
\end{tabular}

Primeira escolha ao pote com a dieta A não difere pelo teste qui-quadrado, e RI pelo teste-t $(\mathrm{P}>0,05)$.

${ }^{\mathrm{a}}$ Primeira escolha ao pote com a dieta B é obtida como $36-\mathrm{n}$.

${ }^{\mathrm{b}} \mathrm{RI}$ : [g ingeridas da dieta $\mathrm{A}$ ou $\mathrm{B} / \mathrm{g}$ totais consumidas $\left.(\mathrm{A}+\mathrm{B})\right] \mathrm{x} 10$.

A avaliação da palatabilidade é importante por medir a atratividade, o consumo e o aceite de um alimento (Tobie et al., 2015). Mas vale ressaltar que esse teste pode ser influenciado por diversos fatores, como os palatabilizantes utilizados e a interação deles com outros ingredientes da composição das dietas (Félix et al., 2012).

\section{CONCLUSÕES}

Não há efeito do período de adaptação sobre a digestibilidade e a EM das dietas, bem como sobre as características fecais em cães, exceto para a amônia fecal, que sofre redução após 10 dias de alimentação. A inclusão de $15 \%$ de casca de soja na dieta reduz a digestibilidade da matéria seca e a energia metabolizável, sem alterar a palatabilidade. Ainda, reduz o $\mathrm{pH}$ e a amônia fecal de cães.

\section{REFERÊNCIAS}

BRUNSGAARD, G.; BACH KNUDSEN, K.E.; EGGUM, B.O. The influence of the period of adaptation on the digestibility of diets containing different types of indigestible polysaccharides in rats. Br. J. Nutr., v.74, p.833-848, 1995.

CAMPBELL, K.L.; CAMPEBELL, J.R. Feeding and nutrition of dogs and cats. in companion animals. Their biology, care, health, and management. 2ed. Upper Saddle River: Pearson Education Inc., 2009. chap.9, p.253-299.
COLE, J.T.; FAHEY G.C.; MERCHEN N.R. et al. Soybean hulls as a dietary fiber source for dogs. J. Anim. Sci., v.77, p.917-924, 1999.

DOG and cat nutrient profiles. Oxford: AAFCO, 2004. (Official Publications of the Association of American Feed Control Officials Incorporated).

FÉLIX, A.P.; CARVALHO, M.P.; ALARÇA, L.G. et al. Effects of the inclusion of carbohydrases and different soybean meals in the diet on palatability, digestibility and faecal characteristics in dogs. Anim. Feed Sci. Tech., v.174, p.182-189, 2012.

GRIFFIN, R.W. Palatability testing: parameters and analysis that influence test conclusions. Petfood Technol., p.187-193, 2003.

GUILLON, F.; CHAMP, M. Structural and physical properties of dietary fibres, and consequences of processing on human physiology. Food Res. Inter., v.33, p.233-245, 2000.

KOPPEL, K.; MONTI, M.; GIBSON, M. et al. The effects of fiber inclusion on pet food sensory characteristics and palatability. Animals, v.5, p.110-125, 2015.

KUZMUK, K.N.; SWANSON, K.S.; TAPPENDEN, K.A. et al. Diet and age affect intestinal morphology and large bowel fermentative end-product concentrations in senior and young adult dogs. J. Nutr., p.19401945, 2005. 
MATEOS, G.G.; JIMÉNEZ, E.M.; SERRANO, M.P. et al. Poultry response to high levels of dietary fiber sources varying in physical and chemical characteristics. J. Appl. Poult. Res., v.21, p.156-174, 2012.

NOOT, H.M.; RIGBY, S.I.; JOHNSON, J.V. et al. Design of digestibility trials for dogs and cats. J. Nutr., v.124, p.2582-2583, 1994.

NUTRIENT requirements of dogs and cats. 2.ed. Washington: National Academies Press, 2006. 450p.

OFFICIAL methods of analysis. 16.ed. Washington: AOAC, 1995.

SABCHUK, T.T.; LOWNDES, F.G.; SCHERAIBER, M. et al. Effect of soya hulls on diet digestibility, palatability, and intestinal gas production in dogs. Anim. Feed Sci. Technol., v.225, p.134-142, 2017.
SÁ-FORTES, C.M.L. Valor nutricional de ingredientes energéticos e protéicos para cães. 2005, 82f. Tese (Doutorado em Zootecnia) Universidade Estadual Paulista, São Paulo.

TOBIE, C.; PÉRON, F.; LAROSE, C. Assessing food preferences in dogs and cats: review of current methods. Animals, v.5, p.126-197, 2015.

WEBER, M.; BISSOT, T.; SERVET, E. et al. A high-protein, high-fiber diet designed for weight loss improves satiety in dogs. J. Vet. Intern. Med., v.21, p.1203-1208, 2007.

YAMKA, R.M.; FRIESEN, K.G.; SCHAKENRAAD, $\mathrm{H}$. The prediction of urine $\mathrm{pH}$ using dietary cations and anions in cats fed dry and wet foods. Intern. J. Appl. Res. Vet. Med., v.4, p. 355-361, 2006.

ZHANG, W.; LIU, D.L.L.; ZANG, J. et al. The effects of dietary fiber level on nutrient digestibility in growing pigs. J. Anim. Sci. Biotechnol., v.4, p.4-17, 2013. 